Note: This is a draft of a paper submitted for publication. Contents of this paper should not be quoted or referred to without permission of the author(s).

Submitted at the Fall 2000 Materials Research Society Meeting

Symposium Proceedings, "Ion Beam Synthesis and Processing of Advanced Materiais"

\title{
Nanophase Composites Produced by Ion Implantation: Properties, Problems, and Potential
}

\author{
A. Meldrum, ' L. A. Boatner, ${ }^{2}$ C. W. White, ${ }^{2}$ and R. F. Haglund, Jr. ${ }^{3}$ \\ 'Department of Physics. University of Alberta, Edmonton, AB T6G Canada \\ ${ }^{2}$ Solid State Division, Oak Ridge National Laboratory, Oak Ridge, TN 37831 \\ ${ }^{3}$ Department of Physics and Astronomy, Vanderbilt University, Nashville, TN 37235
}

\begin{abstract}
The submitted manuscript has been authored by a contractor of the U.S. Government under contract No. DE-AC05-00OR22725. Accordingly, the U.S. Government retains a nonexclusive, royaltyiree licence to nublish or reproduce the pubtished iom of this contribution, or this contribution or allow others to do s). for U.S. Government purposes."
\end{abstract}

Prepared by the SOLID STATE DIVISION OAK RIDGE NATIONAL LABORATORY

Managed by

UT-BATTELLE, LLC, for the U.S. DEPARTMENT OF ENERGY

Under Contract No. DE-AC05-00OR22725 


\title{
Nanophase Composites Produced by lon Implantation: Properties, Problems, and Potential
}

\author{
A. Meldrum \\ Department of Physics. University of Alberta. Edmonton. AB T6G 2J1 Canada \\ L. A. Boatner and C. W. White \\ Solid State Division. Oak Ridge National Laboratory, Oak Ridge IN 37831 U.S.A.
}

\author{
R. F. Haglund. Jr. \\ Department of Physics and Astronomy. Vanderbilt University, Nashville TN 37235 U.S.A.
}

\begin{abstract}
Ion implantation has become it versatile and powerful technique for synthesizing nanometerscale clusters and crystals embedded in the near-surface region of a variety of hosts. The resulting nanocomposite materials often show unique optical. magnetic, and electronic properties. Here we review some of the principal features of this nanophase materials synthesis technique and discuss the outstanding experimental difficulties that currently hamper the development of devices based on the many unique properties of these nanocomposite materials. Possible solutions to these problems and future research directions are discussed. The following is a summary paper that is partially based on a recent invited article by the above authors to appear Advanced Materials.
\end{abstract}

\section{Overview}

Nanophase materials are frequently characterized by novel properties that can be significantly different from those of the corresponding bulk phase. As precipitated nanocrystals (NCs) are formed on ever decreasing length scales. the differences between the bulk and small-particle properties become increasingly pronounced. These differences have stimulated a growing worldwide effort that cuts across many disciplines and research areas that emphasizes the synthesis and characterization of an increasingly wide variety of nanocomposite materials. The practical motivation for this intense research effort derives both from the fundamental characteristics of smail particles as well as the numerous potential applications of these materials. particularly in the areas of optical devices, micromechanical devices, and information storage. The novel properties of nanophase particles are dominated by two major effects. These are. first. the increasing relative significance of the surface-energy contributions associated with the larger surface-to-volume ratio of small particles and, second, the unique characteristics of electrons in confined systems. The first effect largely determines the physical properties of the particles or the nanocomposite (e.g. melting points. solid phase transitions, bulk modulus). Both the surface properties and electron confinement combine to produce novel electronic properties that can be manifested in a wide range of effects, such as a large nonlinear optical susceptibility, intense photoluminescence. altered band structures, and superparamagnetism, to name but a few.

Many experimental techniques have been developed for synthesizing various types of nanocomposite materials. Ion implantation was first used for this purpose in the 1970 s to form 
Ag and Au nanocrystals embedded in silica ylass." It that time. however. there were no obvious applications for such nanocomposites. so it was not untii the 1990 s that ion implantation became in important and widely used research techniaue for synthesizing nanocomposite materials. Today. over wo dozen research groups on tive continents are atively involved in the synthesis and characterization of nanocomposites formed by ion implantation. and entire sessions or symposia at major conferences ure now devoted to this topic.

The increasing popularity of the ion implantation technique is due in part to its

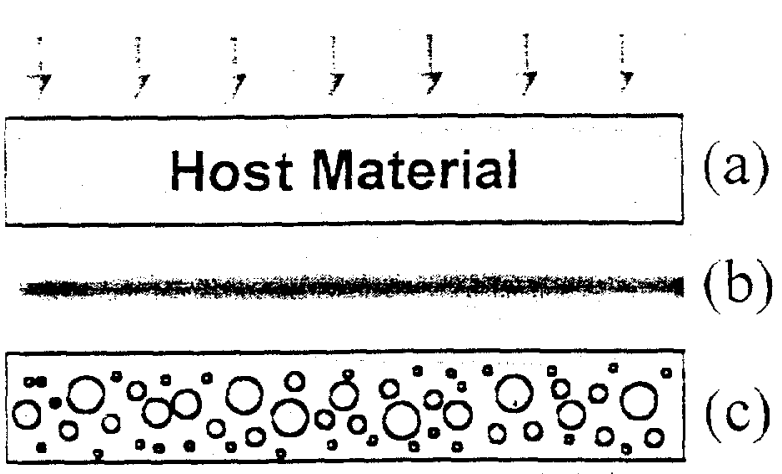

Fig. 1. Schematic of the ion implantation technique. In (a), a chosen host matcrial is implanted with energetic ions (arrows). producing a supersaturated solid solution in the near-surface region (b). In (c), subsequent thermal processing has caused the implanted material to precipitate as jiscrete particles.

rersatility and flexibility. In this lechnique. a selected host material (frequently an insulating ceramic) is injected with energetic ions that are accelerated from a few tens to a few thousand kilovolts. High-dose implantation can create a solid state supersaturation of the implanted ions in a layer extending from the specimen surface to a depth of several tens to hundreds of nanometers. Subsequent thermal processing or further irradiation can, depending on the specific host/nanoparticle solid state chemistry. induce the implanted material to precipitate as discrete nanoparticles (Fig. 1). The versatility of the implantation technique arises from the fact that essentially any element in the periodic table can be implanted into virtually any selected host material. This versatility and the various possible combinations of implanted ions allow for an extremely large range of polential nanoparticle-host combinations. Useful properties of two or more precipitated phases can he combined into one well-defined. integrated structure: and the important physical properties of the nanocomposite can be optimized for a particular application by controlling the concentration and arerage size of the precipitates. Depending on the physical and chemical properties of the host material. nanocomposites formed by ion implantation can be durable since they are formed below the host surface and are thus protected from the surrounding environment. The average precipitate size can be controlled by varying the concentration of implanted ions (e.g., by selecting the appropriate dose, dose rate, and energy). Finally, ion implantation is now widely cmployed in the semiconductor industry for doping silicon wafers, and therefore. it constitutes a materials technology that is already established in the commercial synthesis and processing of materials with microscopic precision and control.

\section{Types of Composites}

Metallic clusters embedded in an insulating host were among the first nanocomposite systems to be tormed by ion implantation." In the early work, it was noted that an extremely high local concentration of precipitates was obtained in a thin layer near the specimen surface. This physical contiguration is quite different from conventional nanocluster composite glasses made from melt processes. where the particles are relatively uniformly dispersed throughout the bulk. Metal-nanocluster composites formed by ion implantation exhibit pronounced optical effects including: 1) absorption due to surface-plasmon resonance, and 2) strong third-order nonlinear 
optical susceptibility. Both classicai and quantum-mechanical effects are at work in these phenomena. The spatial continement of the metailic electrons by the insulating host produces an eninanced electromagnetic ficld due to the large dipole moment induced by the optical field. In addition. for very small nanourysals (with diameters less than approximately $10 \mathrm{~nm}$ ) the continement of the electron wave runctions in either the initial or final states to a volume much smaller than their bulk mean tree path produces an additional contribution to the electric susceptibility.

Metal-cluster nanocomposites formed by ion implantation have excellent nonlinear optical properties. The experimentally determined value of the effective nonlinear optical susceptibility $\left(\chi^{i \prime}\right)$ is generally higher by a lactor of $10^{3}$ over conventional quenched nanocomposite glasses. and the response times are on the order of picoseconds. Several authors have suggested that these materials may have potential applications in all-optical memory or optical switching devices [3.4]. However, certain difficulties remain to be addressed before these types of device applications can be realized. A major problem is that the linear absorption and the nonlinear optical susceptibility both peak at the surface plasmon resonance (SPR) frequency. In addition to decreasing optical transmission. the high SPR absorption coefficient can produce an associated long-lived thermal component in the noniinear response. although the thermal relaxation problem may be decreased if sufficienty thort oprical puises are used [5]. One way to solve some of these problems is to have a extremely narrow size distribution of the smallest particles - enhancing

Table 1. Catalogue of semiconductor nanocrystals produced by ion implantation.

\begin{tabular}{|c|c|c|c|}
\hline \multirow[t]{2}{*}{ Nanocrystal } & \multicolumn{3}{|c|}{ Host Material } \\
\hline & $\mathrm{Al}_{2} \mathrm{O}_{3}$ & $\mathrm{Si}$ & $\mathrm{SiO}_{2}$ \\
\hline $\mathrm{CdS}$ & $P$ & Fo & $p$ \\
\hline $\mathrm{CdSe}$ & $B$ & $B$ & $P$ \\
\hline $\mathrm{CdTe}$ & & \& & $\beta$ \\
\hline GaAs & Fo & Fo & $F$ \\
\hline GaN & $z$ & & \\
\hline $\mathrm{GaP}$ & Fo & F & Fo \\
\hline Ge & Fo & & Fo \\
\hline InAs & & 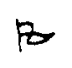 & Fo \\
\hline $\operatorname{InP}$ & & Fo & 8 \\
\hline $\mathrm{PbS}$ & B & & ß \\
\hline $\mathrm{PbTe}$ & Fo & & \\
\hline $\mathrm{Si}$ & B & & Po \\
\hline $\mathrm{ZnS}$ & Fo & F & Fo \\
\hline $\mathrm{ZnSe}$ & & & 8 \\
\hline $\mathrm{ZnTe}$ & & & p \\
\hline $\mathrm{VO}_{2}$ & $B$ & & 8 \\
\hline
\end{tabular}

$\chi^{(3)}{ }_{e f f}$ and decreasing the SPR absorption.

Semiconductor nanocomposites formed by ion implantation have also been widely investigated, due to their potential applications in as lightemitting diodes [6] and single-electron transistors [7]. They have potential applications in nonvolatile memory [8], nanocrystal gate oxide [9], and "smart" temperature- and lightsensing devices [10]. A large number of singleelement and compound nanoclusters have been formed in a variety of insuiating host materials (Table 1). and prototype devices have been built [11]. These materials have many unique properties. for example, $\mathrm{Si} \mathrm{NCs}$ in $\mathrm{SiO}_{2}$ are strongly luminescent (although the origin of the light emission is still debated), CdS nanocrystals show clear evidence for quantum-confinementinduced bandgap shifts, and $\mathrm{VO}_{2}$ precipitates switch from the semiconducting state to the metallic state over the temperature range 340 $360 \mathrm{~K}$, suggesting applications as optical switches or "smart" temperature sensors [12].

Despite these recent successes, several problems currently hinder the development of functional semiconductor-NC-based devices. Particle size distributions are too broad and the 
microstructural relationsnips between the precipitates and the host material are orten complex. These problems are more suere for compound nanoparticles. The broad size distribution is a particular problem for semiconductor nanocomposites. since the eiectronic properties of the precipitates are so strongly dependenc un particle size. The band structure of the composite is thus "blurred" over a range of precipitate sizes. Complex microstructures. 100. are a problem since it becomes difficut to determine the origin of certain types of optical signals. For example, particles may form as hollow shells. thus increasing the internal surtace area and possibly enhancing surface effects [13]. Whe may he blanketed by reaction rims [13]. or they may have non-uniform compositions $[1+.15]$

Ferromagnetic nanoparticles formed by ion implantation also show several unique properties. Ion implantation has the advantage that the precipitates can be made to crystallographically align with the host material. so that the magnetic "easy" axes of the precipitates are parallel. This type of alignment has been demonstrated for Fe and Co particles emedded in transparent crystalline hosts $[16,17]$. As a result of precipitate alignment and the near two-dimensional specimen geometry. the magnetic hysteresis is strongly dependent on the orientation of the magnetic field wh respect to the specimen. The hysteresis and coercivity can. in fact. be controlled by subsequent implantation of $\mathrm{Pt}$ or $\mathrm{X}_{\mathrm{i}}$ into the layer containing the nanoparticles [17]. These initial results are encouraging: however. hr single-particle-per-bit magnetic recording. the precipitates must also be magnetically isolated similar in size. and their position and spacing must be controlled [18]. Control over the size. position. and spacing of the particles is a problem that is yet to be solved.

\section{Outstanding Difficulties}

Ion implantation has been used to torm a wide range of nanoparticle-host combinations (e.g., see Table 1). Clearly the versatility and thexibility of ion implantation for producing many types of nanocomposites has been conclusively demonstrated by many research groups. Control over average size. orientation. morphology crystal structure. and composition has been demonstrated in various experiments. That these nanocomposites have interesting and often unique properties is clear: however. development of actual devices has been hampered by several outstanding experimental difficulties associated with the implantation technique.

First and foremost of these difficulties is the control over the size distribution of the precipitates. Since the properties of nanopurticles are dependent on their size. for many types of applications it is essential to obtain an extremely narrow size distribution. By the nature of ion beam-solid interactions. injecting mono-cnergetic ions into a solid material produces a roughly Gaussian distribution of implanted ions. This is due principally to ion straggling (the statistical nature of ion-target collisions): however. irradiation-induced modifications to the host material (e.g., changes in density. crystal structure. etc.) and energy variations within the ion beam may also affect the distribution of implanted material. Due to the non-uniform implant profile, larger particles often form where the injected ion concentration is the highest (as depicted in Fig. 1). Vucleation and growth of pricipitates during a thermal processing step frequently compounds the problem. Conventional Ostwald ripening processes generally do not occur due to the complex nature of the composite: Radiation damage. the presence of a specimen surface. and interactions and reactions involving the implanted material and the host all serve to complicate the microstructures and size distributions. In short. ion implantation combined with thermal 
processing has not - hus lat - produced sutably narrow size distributions for many types of Levice applications.

i second requirement for appitications sich as magneric data recording is control ofer the spatial bocation of the precipitates. In impiantation has the advantage vier conventional quenched nanocomposite glasses in that the wepth and depth distribution or particles can be controlled. and additionaily. nanocomposites consisting of several types of clusters located at different layer deptins can be produced. Howcer. ton implantation is currently unable to control the location of the precipitates within the implanted layer. In many types of appications le.g.. magnetic recording. optical memory. ches. roular spacing of the precipitates is of critical importance.

A third difficulty is related to compositional uniformity and nanocrystal-host interface states. Although compositional uniformity generally is not a problem in single-element NCs. compound multi-element nanoparticles can have significant non-uniformities in terms of their composition and structure [13]. Nanocrystals prepared by chemical techniques are passivated with selected organic ligands and the nature of the interface is fairly well understood: however. the effects of the particle-host interface prticularly in the optical properties of embedded nanocrystals cormed by ion implantation. is inughe to be important but is poorly understood. In some cases e.g.. $\mathrm{Si} \mathrm{NCS}$ in $\mathrm{SiO}_{2}$ ), the incuriace is probabiy controls the strong light emission [19.20], but the exact mechanism is still under in estigation.

Another difficulty relates to the means by which the nanocomposites are synthesized and studied. Currently. specimens must removed from the implanter for thermal processing and characterization - i.e.. a serial technique. For example. as discussed above, recent work has shown that the magnetic propertics of $C o$ precipitates in a sapphire host can be controlled by subsequent implantation of Pt as . $\mathrm{X}$. l fowever. to perform these experiments requires specimens to be re-implanted many limes. nmetimes re-annealed. and the specimens are exposed to atmosphere on many occasions. Thus. the experiments are laborious and time consuming, may be non-reproducible. and pussibly. interesting physics could be missed - for example at intermediate ion doses. The possibility of combining ion implantation with in-situ specimen characterization will be explores below

New studies are clearly needed that fincus on innovative ideas and solutions relating to some of these outstanding problems. Much work has been done in the last decade to clearly demonstrate the novel properties of these composites. and to show that an extremely large number of nanoparticle compositions cun he tormed in an almost unlimited number of host materials. The Jirection is now towards optimization of these materials and finding solutions to the outstanding difficulties that hamper the derclupment of these nanocomposites in various types of devices. This will involve several factors. including the development of newer implantation techniques (e.g.. focused ion beams). the combination of ion implantation with other techniques such as pulsed laser deposition (c.s.. the new Vanderbilt system) or thin film growth (either by implanting thin films or by using lithographic tilms as masks), and the development of in-situ ion implantation facilities such as that recently funded at the University of Alberta. These kinds of new research directions and their potential benefits will be discussed in the following section. 


\section{Potential solutions and Future Research Directions}

\section{Focused ion beams}

One idea to overcome particle sze and spacing difficulties is to selectively impian specitic areas of a specimen using a focused ion heam IFIBi. This is an emerging technolog! in ahich a set of electromagnetic lenses is used to collimate and focus an ion beam into a narrow spot isumewhat akin to electron lenses in electron microscopy). The most modern instruments claim minimum spot sizes as small as about ten nanometers. Commercial FIBs are primarily restricted to $35 \mathrm{kV}$ (ia sources. which is currently a major limitation w their use as implanters. At this low energy, sputterng is the major difficuity since the implanted ions cannot he injected sufficiently deep into the hat. wh the beam acts more like an "ion drill" (c... lig. 2) (ja may have some applications (e.g.. it may he possible to make GaAs particles in specimens pre-implanted with As); however. the applications at a (ia lon beam for implantation are limited.

In recent experiments [2!], a cicused ion beam was used to implant an array of spots in a silicon host. The FIB was programmed to inject a $1 \mathrm{~mm}^{2}$ area with $2 \mathrm{x}$ $10^{i 1)}$ ionsispot at a spot spacing of $10 \mu \mathrm{m}$. The regions

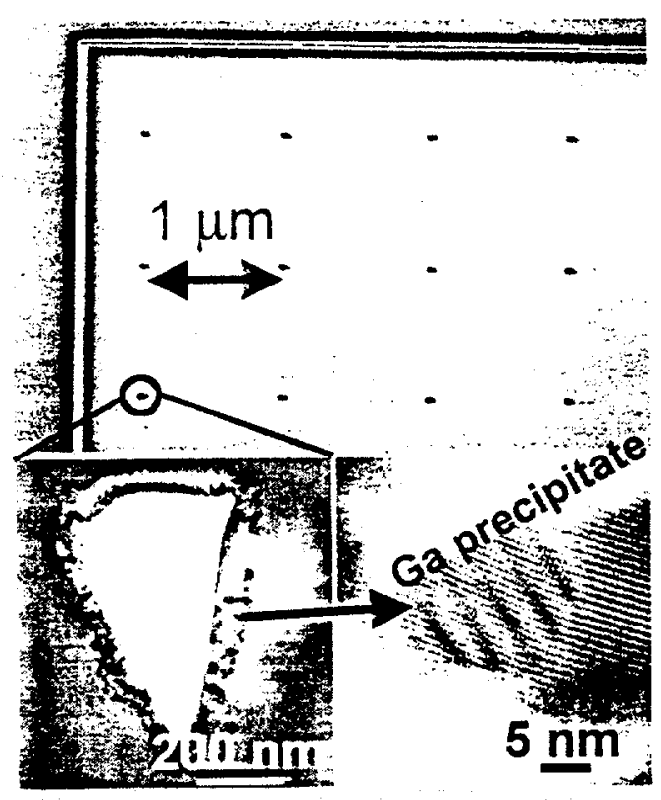

Fig. 2. Top: backscattered SEM image showing an array of holes "drilled" in single-crystal silicon with a focused $35 \mathrm{kV}$ Ga ion beam. Bottom left: TEM image showing a laver of precipitates around the edges of one of the holes. One of the precipitates is magnified in the bottom right image.

around the edges of the drilled holes do contain fine Ga precipitates (Fig. 2). This proof-ofprinciple experiment conclusively demonstrated that the FIB can produce ordered spots on a substrate. and that it is even pussible to produce precipitates around the edges of the ion-drilled regions. The results suggestad that commercial FIB technology is promising technique. but it has not yet reached the level of suphistication required to provide the higher energies and a wider range of ions necessary for its usc in implantation. Additionally. FIB implantation is a serial technique. so even if ion source difficulties are overcome, implantation of relatively large areas will still be a slow and costly procedure. For example. the specimen described above contained one 10.000 individual implanted spots. requiring many hours of implantation time. To synthesize a larger specimen with a higher concentration of particles would. at the current level of technology, require a prohibitively long implantation times.

\section{Combination techniques}

One particularly interesting area is the combination of thin film growth with ion implantation. The ability to create high quality waveguide films. for example. leads to the possibility of implanting such materials to create an enhanced optical response. In one idea. it may be possible to create buried layers of particles within a thin waveguide film. The formation of nanoparticles 
athin a waveguide tilm has ben. forecon yours. the focus of intense researcin storts $1 .$. . se Ret. al) The optical rasponse of the anopartebs ahen measured "adge-on". should te uriss ul masnitude stronger than conventional pane :arpondicular measurements. Obriously many hiticultes may be encountered. such as the elicets 11 implantation on the optical properties of the him. ne clfects of the intertace te.g.. the intertace may as a favorable nucleation site for the precipitutesi. and chemical reactions between the film and the impianted material. A new state-ot-the-art LIV thin film deposition system is being buile at the liniversity of Alberta that will find considerable usc this research area.

In another application combining !h tims and ion impiantation. it may be possinic 11 us onventional lithographic techniques a intiksise films or membranes with regularly pacid holis. A selected substrate couid then be implantud througn the holes to create an ordered array of nanoparticles (Fig. 3). This technique has been highly vaccsstul in producing ion-beam-patterned magnetic i!ms. Fir sxample. the Orsay group synthesized - -114)-mm-hick silica films using a conventional lithographic schnique [23]. These masks were located dirce!! " "il the a specimen for ion implantation (Fig. 4). Mter inaplancation of IIe ions. the mask was removed and the magnetic properties of the resulting lim lie patterned according to the mask shape.

Here. We envision the possibility using a similar technique to create ordered arrays wi nanoparticles. The mask requirements will be inrly sure. The film would have to be thick enough sis stop the ions. and durable enough to withstand high-dose implantation without breaking. disintegrating. or swelling. The size of the holes and the thermal processing conditions will probably datermine whether a single particle would form at each location. or a group of particlis. 1t. for cxample. this technique could be used to synthesize a regular array of embedded nanoparticles. this would overcome a major obstacle currently limiting the potential application of such materials in magnetic recording and optical memory. This is a research idea that is wine wiective of the nanoparticle research being conducted through collaborations between OR.VL. Vinuarbilt. and the University of Alberta.

\section{In-situ techniques}

In-sinn experimental techniques can be useful for optimizing specific microstructural or electronic properties of ion-implanted nanocomposites. In one idea. we have attempted to use insitu TEM techniques to monitor specimens during implantation and thermal processing. The 
IVEM-Tandem Facility at Argonne National Laboratory consists of a $650 \mathrm{keV}$ NEC accelerator interfaced to a Hitachi H9000NAR transmission electron microscope [24]. This facility has traditionally been used for in-situ radiation-damage experiments; however. by decreasing the ion energy it is readily possible to implant a TEM specimen. In one example illustrating the flexibility of the ion implantation technique, both ferromagnetic and Type I superconducting particles were produced in the same sample. A sample containing pre-existing $\mathrm{Ni}$ nanocrystals was implanted with $\mathrm{Pb}$ ions in-situ directly in the TEM. Two distinct and separate types of nanoparticles were formed: i.e., relatively large $\mathrm{Pb}$ precipitates and small magnetic Ni particles (Fig. 5). The implanted $\mathrm{Pb}$ concentration was increased until radius of the $\mathrm{Pb}$ precipitates was larger than the coherence length for bulk $\mathrm{Pb}$. This represents one example where in-situ ion implantation can be used to directly monitor the specimen evolution during the implantation and thermal processing steps. The experiment also illustrates that ion implantation can be used to create two distinct types of particles with different properties at the same layer depth within a single specimen. In this specimen, the ferromagnetic Ni precipitates might be expected to modify the correlated-electron properties of the $\mathrm{Pb}$ precipitates at cryogenic temperatures. Magnetic measurements of this specimen are ongoing.

In-situ specimen characterization is also important. At the University of Alberta we have recently obtained funding to build a modified endstation on our Varian CF3000 ion implanter. This endstation will be interfaced to an independent photoluminescence system consisting of a deuterium lamp and associated lenses, CCD camera, and computer hardware. A second port will interface to a picosecond pulsed Ti:sapphire laser for pump-probe reflectivity measurements. We hope to be able to measure various opto-electronic properties as the specimen is implanted (by stopping the ion beam) or during subsequent in-situ thermal or irradiation-induced nucleation and growth. The ability to directly measure the optical properties in-situ should greatly assist in optimizing the desired properties of the nanocomposite, and we also anticipate numerous other applications in non-nanocrystal-related research.

\section{New nucleation techniques and low-energy implantation}

Non-thermal nucleation and growth techniques may be able to produce narrower size distributions than more conventional thermal tech-niques. In one example, a specimen of $\mathrm{SiO}_{2}$ glass was implanted with $\mathrm{Zn}+\mathrm{S}$ at cryogenic tempcratures, to prevent nucleation during the implantation step. Subsequent room-temperature electron irradiation produced a narrow size distribution (by ion implantation standards) of $\mathrm{ZnS}$ nanocrystals embedded in the $\mathrm{SiO}_{2}$ (Fig. 6). Thermal nucleation, in contrast, produces an extremely wide - almost bimodal - size distribution of precipitates. Electronic energy loss processes were dominantly responsible for particle 
auclation [15]. More rocenty he Ursay group has demonstrated means by which the rucieation and growth stages cur be comporaly suparated. so that both processes are independent

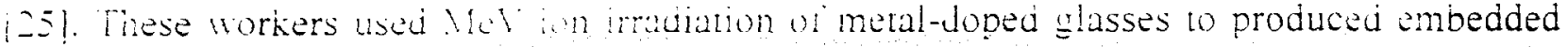
metailic precipitates. Similar in the ruts in Fig. o. an electronic energy loss mechanism was vunci to control the nuckeaton sage ther groups are also actively involved in non-thermal aucieation techniques. for example. Ahe Alabama A\&M and ORNL groups have investigated the properties of specimens in whin nigh-energy ion irradiation induced nanoparticle formation in pre-implanted specimens [26]. (Merail. these techniques do seem to offer hope for considerably narrowing the size distributions. Whough it is rery doubtful that the type of monolayer control demonstrated in chemical cechiques will be achieved using irradiation-induced nucleation and growth protocols.

Low-energy (E)am $\leq 50 \mathrm{hal}$ ) ion impiantation also offers signiticant potential for control of nanocrystal size in layered structures. At low energy and high beam current densily. deposition time and range straggling are minimiaci. while depth uniformity is simultaneously maximized. Post-deposition laser ur thermal annealing treatments can be carried out with higher efficiency and at luwer temperature. becausi implantation-induced end-utrange damage is also reduced. Enhanced control ot

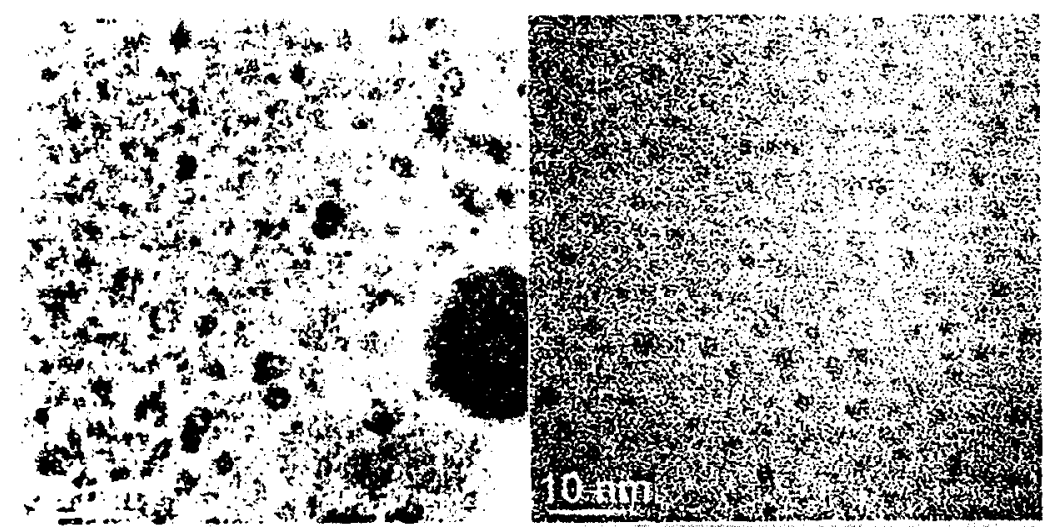

Fig. 6. ZnS nanocrystals grown by ion implantation of $\mathrm{Zn+S}$ followed by thermal processing (left) or electron-irradiationinduced nucleation and growth (right). Both specimens were impianted under similar conditions and to similar doses (a complete descriprion of implantation and annealing conditions is siven in Ref. 15). Irradiation-induced nucleation and growth roduces a considerably narrower size distribution.

\section{nanocrystal size was recent!}

demonstrated for a single-laver array uf $\mathrm{Sn}$ nanocrystals (diameter $4.8=1.0 \mathrm{~nm}$ ) in $\mathrm{SiO}_{2}$ film by impianting at $10 \mathrm{keV}$. io duses at arder $510^{i 5}$ ions $\mathrm{cm}^{-2}$ [27]. This approach solves many problems in present blanket impiantation experiments. but still exhibits one major weakness the random lateral spacing of the manocrystals.

\section{Conclusions}

Ion implantation is a versatile and tlexible technique for creating a wide range of nanocomposite materials with many promising applications. Prototype devices have been built and the results are encouraging. Recent divilopments in the areas of noble metal. semiconductor. and ferromagnetic nanoparticles were liscussed. Despite the many advantages and unique properties of these nanocomposites. suveral problems may hamper the future development of actual devices. The most critical of these are control over the size distribution and spacing of the precipitates. Future research directions and possible means to solve these problems were discussed. The combination of iun implantation with thin film technology (implantation of thin films, development of durable lithographic masks) appears to be one of the most promising 


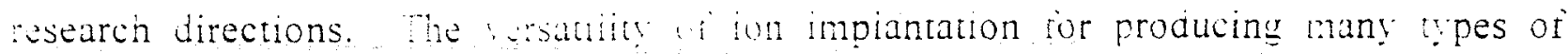
nanocomposites has been civary cutabisined at this juncture: the critical research direction is now towards tinding solutions tor the oustanding pronlems and the ontimization of the unique properties of these materiais.

\section{Acknowledgments}

The in-situ ion implantation experment was carried out at the IVEM Faciiity. Argonne National Laboratory. Research at the Caversity of Aberta is supported by the NSERC. Canadia. Work at ORNL is sponsored by the LS. Dipartment of Energy under contract DE-AC05-00OR22725 with Oak Ridge National Laboratory (ORNL). managed by UT-Battelle. LLC. C. W. White and L. A. Boatner also received support trom the Laboratory Directed Research \& Development Program of ORNL. Nanostructure research at Vanderbilt is supported by a Major Research Instrumentation grant from thi Vational Science Foundation (DMR-9871234). the Southeastern Iniversities Research Association and the Vanderbilt University College of Arts and Science.

\section{References}

[1] A. Meldrum. R.F. Haglund. Ir. I...1. Boaner. and C.W. White. Advanced Materiais (in review).

[2] G. W. Amold and J. A. Borders. J. Appl. Phys. 48. 1488 (1977).

[3] L. Yang, D. H. Osborne. R. F. Haglund Jr.. R. H. Magruder. C. W. White. R. A Zuhr. and H. Hosono. Appl. Phys. A 62. 千(1) (1996).

[4] R. F. Haglund Jr.. L. Yang. R. H. Magruder III. C. W. White. R. A. Zuhr. L. Yang. R. Dorsinville. and R. R. Mano. Nucl. Instr. Meth. B91. 493 (1994).

[5] V. Halté. J. Guille. J.-C. Marke. I. Perakis. and J.-Y. Bigot. Phys. Rev. B 60. 11738 (1999).

[6] V. L. Colvin. M. C. Schlimp. and A. P. Alivisatos. Nature 370. 354 (1994).

[7] D. L. Klein. R. Roth. A. K. L. Lim. A. P. Alivisatos, and P. L. McEuen. Nature 389. 699 (1997).

[8] L. Guo. E. Leobandung. and S. Y. Chou. Appl. Phys. Lett. 70. 850 (1997).

[9] S. Coffa. E. Castagna. C. Bungiurno. and D. Patti. Nucl. Instr. Meth. (in press).

[10] L. A. Gea. J. D. Budai. and L. .. Boamer. J. Mater. Res. 14. 2602 (1999).

[11]N. Lalic and J. Linnros. J. Lumin, 80. 263 (1999).

[12] I. 1. Gea. J. D. Budai. and I. I. Boaner. J. Mater. Res. 14. 2602 (1999).

[13] A. Meldrum. L. A. Boatner. C. WI. White. and R. C. Ewing. Mat. Res. Innovat. 3. 190 (2000).

[14] E. Cattaruzza. G. Battaglin. R. Polloni. T. Cesca. F. Gonella. G. Mattei. C. Maurizio. P. Mazzoidi. F. D Acapito. F. Zuntone, and R. Bertoncello. Nucl. Instr. Meth. B148. 1007 (1999).

[15] A. Meldrum. E. Sonder. R. A. Luhr. I. M. Anderson. J. D. Budai. C. W. White. L. A. Boatner. and D. O. Hendurson. J. Mater. Res. 14. 4+89 (1999).

[16] S. Honda. F. A. Modine A. Meldrum. J. D. Budai. T. E. Haynes. and L. A. Boatner. Appl. Phys. Lett. 77.711 (2000).

[17] S. Honda. F. A. Modine. T. E. Haynes. A. Meldrum. J. D. Budai. K. J. Song. J. R. Thompson. and L. A. Boatner. Mat. Res. Soc. Symp. Proc. 581. 71 (2000). 


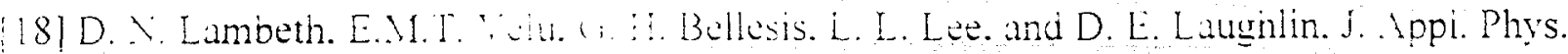
$79 .+496(1996)$

191 C. W. White. S. P. Withrus \& Mekum. I. D. Budai. D. M. Hembree. J. G. Zhu. D. O.

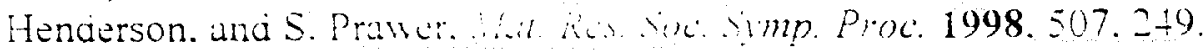

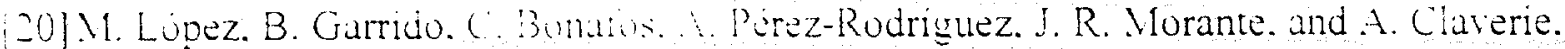
Vici. Hinstr. Weth $B$ in prosst.

[21]R. A. Zuhr. J. D. Budai. P. (i. Datshus. (C. M. Egert. A. Meldrum. K. A. Thomas. C. W. White. L. C. Feldman. W. Smonet and K-H. Heinig. Mater. Res. Soc. Symp. Proc. $\mathbf{5 3 6 . 2 5 1}$ (1999).

[22] R. F. Hagiund. Jr. In Uprics on imail Particles. Surfaces and Interfaces. edited by R.E. Hummel and Peter Wißmulin (Bocu Raton. FL: CRC Press. 1997) 191-231.

[23] T. Devolder. C. Chappert. Y Vathet. H. Bernas. Y. Chen. J. P. Janet. and J. Ferré. J. Appl. Phys. 87. 8671 (2000)

[2+] C. W. Allen and E.A. Ryan. Yat. Res. Soc. Symp. Proc. 439. 277 (1997).

$25]$ E. Valentin. H. Bernas. ( R. Revilcau. and F. Creuzet. Nucl. Instr. Meth (in press).

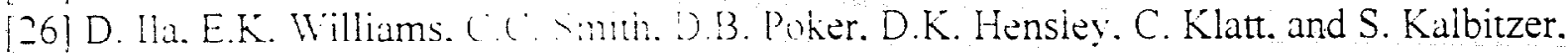
Vucl. Instr. Meth. Phys Ris. 3148.11211999$)$.

[27] A. Nakajima. T. Futatsu!! ` Gigriguchi and X. Yokoyama, Appl. Phys. Lett. 71.3652 (1997) 